\title{
Isometric Differentiable Functions on Real Normed Space ${ }^{1}$
}

\author{
Yuichi Futa \\ Japan Advanced Institute \\ of Science and Technology \\ Ishikawa, Japan
}

\author{
Noboru Endou \\ Gifu National College of Technology \\ Gifu, Japan
}

\author{
Yasunari Shidama \\ Shinshu University \\ Nagano, Japan
}

Summary. In this article, we formalize isometric differentiable functions on real normed space [17, and their properties.

MSC: 58C20 46G05 03B35

Keywords: isometric differentiable function

MML identifier: NDIFF_7, version: 8.1.02 5.22.1194

The notation and terminology used in this paper have been introduced in the following articles: [3], [2], 8], [4], [5], [18, [10], [1], [19], 14, [16], 1], 6], 9], [15], 23], 24], 21], 22], [13], 25], and [7].

\section{Preliminaries}

From now on $S, T, W, Y$ denote real normed spaces, $f, f_{1}, f_{2}$ denote partial functions from $S$ to $T, Z$ denotes a subset of $S$, and $i, n$ denote natural numbers.

Now we state the propositions:

(1) Let us consider a set $X$ and functions $I, f$. Then $(f\lceil X) \cdot I=(f$. $I)\left\lceil I^{-1}(X)\right.$.

(2) Let us consider real normed spaces $S, T$, a linear operator $L$ from $S$ into $T$, and points $x, y$ of $S$. Then $L(x)-L(y)=L(x-y)$.

\footnotetext{
${ }^{1}$ This work was supported by JSPS KAKENHI 23500029 and 22300285. 
(3) Let us consider real normed spaces $X, Y, W$, a function $I$ from $X$ into $Y$, and partial functions $f_{1}, f_{2}$ from $Y$ to $W$. Then

(i) $\left(f_{1}+f_{2}\right) \cdot I=f_{1} \cdot I+f_{2} \cdot I$, and

(ii) $\left(f_{1}-f_{2}\right) \cdot I=f_{1} \cdot I-f_{2} \cdot I$.

Proof: Set $D_{1}=$ the carrier of $X$. For every element $s$ of $D_{1}, s \in$ $\operatorname{dom}\left(\left(f_{1}+f_{2}\right) \cdot I\right)$ iff $s \in \operatorname{dom}\left(f_{1} \cdot I+f_{2} \cdot I\right)$ by [4, (11)]. For every element $z$ of $D_{1}$ such that $z \in \operatorname{dom}\left(\left(f_{1}+f_{2}\right) \cdot I\right)$ holds $\left(\left(f_{1}+f_{2}\right) \cdot I\right)(z)=\left(f_{1} \cdot I+f_{2} \cdot I\right)(z)$ by [4, (11), (12)]. For every element $s$ of $D_{1}, s \in \operatorname{dom}\left(\left(f_{1}-f_{2}\right) \cdot I\right)$ iff $s \in \operatorname{dom}\left(f_{1} \cdot I-f_{2} \cdot I\right)$ by [4, (11)]. For every element $z$ of $D_{1}$ such that $z \in \operatorname{dom}\left(\left(f_{1}-f_{2}\right) \cdot I\right)$ holds $\left(\left(f_{1}-f_{2}\right) \cdot I\right)(z)=\left(f_{1} \cdot I-f_{2} \cdot I\right)(z)$ by [4, (11), (12)].

(4) Let us consider real normed spaces $X, Y, W$, a function $I$ from $X$ into $Y$, a partial function $f$ from $Y$ to $W$, and a real number $r$. Then $r \cdot(f \cdot I)=$ $(r \cdot f) \cdot I$. Proof: Set $D_{1}=$ the carrier of $X$. For every element $s$ of $D_{1}$, $s \in \operatorname{dom}((r \cdot f) \cdot I)$ iff $s \in \operatorname{dom}(f \cdot I)$ by [4, (11)]. For every element $s$ of $D_{1}, s \in \operatorname{dom}((r \cdot f) \cdot I)$ iff $I(s) \in \operatorname{dom}(r \cdot f)$ by [4, (11)]. For every element $z$ of $D_{1}$ such that $z \in \operatorname{dom}(r \cdot(f \cdot I))$ holds $(r \cdot(f \cdot I))(z)=((r \cdot f) \cdot I)(z)$ by [4, (12)].

(5) Let us consider a partial function $f$ from $T$ to $W$, a function $g$ from $S$ into $T$, and a point $x$ of $S$. Suppose

(i) $x \in \operatorname{dom} g$, and

(ii) $g_{x} \in \operatorname{dom} f$, and

(iii) $g$ is continuous in $x$, and

(iv) $f$ is continuous in $g_{x}$.

Then $f \cdot g$ is continuous in $x$. Proof: Set $h=f \cdot g$. For every real number $r$ such that $0<r$ there exists a real number $s$ such that $0<s$ and for every point $x_{1}$ of $S$ such that $x_{1} \in \operatorname{dom} h$ and $\left\|x_{1}-x\right\|<s$ holds $\left\|h_{x_{1}}-h_{x}\right\|<r$ by [14, (7)], [12, (3), (4)].

Let $X, Y$ be real normed spaces and $x$ be an element of $X \times Y$. The functor reproj1 $(x)$ yielding a function from $X$ into $X \times Y$ is defined by

(Def. 1) Let us consider an element $r$ of $X$. Then $i t(r)=\left\langle r, x_{\mathbf{2}}\right\rangle$.

The functor reproj2 $(x)$ yielding a function from $Y$ into $X \times Y$ is defined by

(Def. 2) Let us consider an element $r$ of $Y$. Then $i t(r)=\left\langle x_{\mathbf{1}}, r\right\rangle$.

\section{ISOMETRIES}

Now we state the propositions:

(6) Let us consider a linear operator $I$ from $S$ into $T$ and a point $x$ of $S$. If $I$ is isometric, then $I$ is continuous in $x$. 
(7) Let us consider real normed spaces $S, T$ and a linear operator $f$ from $S$ into $T$. Then $f$ is isometric if and only if for every element $x$ of $S$, $\|f(x)\|=\|x\|$. The theorem is a consequence of (2).

(8) Let us consider a linear operator $I$ from $S$ into $T$ and a subset $Z$ of $S$. If $I$ is isometric, then $I$ is continuous on $Z$. The theorem is a consequence of $(6)$.

(9) Let us consider a linear operator $I$ from $S$ into $T$. Suppose $I$ is one-toone, onto, and isometric. Then there exists a linear operator $J$ from $T$ into $S$ such that

(i) $J=I^{-1}$, and

(ii) $J$ is one-to-one, onto, and isometric.

The theorem is a consequence of (7). Proof: Reconsider $J=I^{-1}$ as a function from $T$ into $S$. For every points $v, w$ of $T, J(v+w)=J(v)+J(w)$ by [5, (113)], 44, (34)]. For every point $v$ of $T$ and for every real number $r$, $J(r \cdot v)=r \cdot J(v)$ by [5, (113)], [4, (34)]. For every point $v$ of $T,\|J(v)\|=\|v\|$ by [5, (113)], [4, (34)].

Let us consider a linear operator $I$ from $S$ into $T$ and a sequence $s_{1}$ of $S$. Now we state the propositions:

(10) If $I$ is isometric and $s_{1}$ is convergent, then $I \cdot s_{1}$ is convergent and $\lim (I$. $\left.s_{1}\right)=I\left(\lim s_{1}\right)$.

(11) If $I$ is one-to-one, onto, and isometric, then $s_{1}$ is convergent iff $I \cdot s_{1}$ is convergent.

Let us consider a linear operator $I$ from $S$ into $T$ and a subset $Z$ of $S$. Now we state the propositions:

(12) If $I$ is one-to-one, onto, and isometric, then $Z$ is closed iff $I^{\circ} Z$ is closed.

(13) If $I$ is one-to-one, onto, and isometric, then $Z$ is open iff $I^{\circ} Z$ is open.

(14) If $I$ is one-to-one, onto, and isometric, then $Z$ is compact iff $I^{\circ} Z$ is compact.

Now we state the propositions:

(15) Let us consider a partial function $f$ from $T$ to $W$ and a linear operator $I$ from $S$ into $T$. Suppose $I$ is one-to-one, onto, and isometric. Let us consider a point $x$ of $S$. Suppose $I(x) \in \operatorname{dom} f$. Then $f \cdot I$ is continuous in $x$ if and only if $f$ is continuous in $I(x)$. The theorem is a consequence of (9), (6), and (5).

(16) Let us consider a partial function $f$ from $T$ to $W$, a linear operator $I$ from $S$ into $T$, and a set $X$. Suppose

(i) $X \subseteq$ the carrier of $T$, and

(ii) $I$ is one-to-one, onto, and isometric. 
Then $f$ is continuous on $X$ if and only if $f \cdot I$ is continuous on $I^{-1}(X)$. The theorem is a consequence of (15) and (1). Proof: For every point $y$ of $T$ such that $y \in X$ holds $f\lceil X$ is continuous in $y$ by [5, (113)], [23, (57)].

Let $X, Y$ be real normed spaces. The functor $\operatorname{IsoCPNrSP}(X, Y)$ yielding a linear operator from $X \times Y$ into $\prod\langle X, Y\rangle$ is defined by

(Def. 3) Let us consider a point $x$ of $X$ and a point $y$ of $Y$. Then $i t(x, y)=\langle x, y\rangle$. Now we state the proposition:

(17) Let us consider real normed spaces $X, Y$. Then ${ }^{0} \prod\langle X, Y\rangle=$ $(\operatorname{IsoCPNrSP}(X, Y))\left(0_{X \times Y}\right)$.

Let $X, Y$ be real normed spaces. Observe that $\operatorname{IsoCPNrSP}(X, Y)$ is one-toone onto and isometric.

Let us note that there exists a linear operator from $X \times Y$ into $\prod\langle X, Y\rangle$ which is one-to-one, onto, and isometric.

Let $f$ be a one-to-one onto isometric linear operator from $X \times Y$ into $\prod\langle X$, $Y\rangle$. Let us note that the functor $f^{-1}$ yields a linear operator from $\Pi\langle X, Y\rangle$ into $X \times Y$. One can verify that $f^{-1}$ is one-to-one onto and isometric as a linear operator from $\prod\langle X, Y\rangle$ into $X \times Y$.

Observe that there exists a linear operator from $\prod\langle X, Y\rangle$ into $X \times Y$ which is one-to-one, onto, and isometric.

Now we state the propositions:

(18) Let us consider real normed spaces $X, Y$, a point $x$ of $X$, and a point $y$ of $Y$. Then $(\operatorname{IsoCPNrSP}(X, Y))^{-1}(\langle x, y\rangle)=\langle x, y\rangle$. Proof: Set $I=$ IsoCPNrSP $(X, Y)$. Set $J=I^{-1}$. For every point $x$ of $X$ and for every point $y$ of $Y, J(\langle x, y\rangle)=\langle x, y\rangle$ by [4, (34)].

(19) Let us consider real normed spaces $X, Y$.

Then $(\operatorname{IsoCPNrSP}(X, Y))^{-1}\left(\prod_{\lfloor\langle X, Y\rangle}\right)=0_{X \times Y}$. The theorem is a consequence of (17).

(20) Let us consider real normed spaces $X, Y$ and a subset $Z$ of $X \times Y$. Then IsoCPNrSP $(X, Y)$ is continuous on $Z$.

(21) Let us consider real normed spaces $X, Y$ and a subset $Z$ of $\prod\langle X, Y\rangle$. Then $(\operatorname{IsoCPNrSP}(X, Y))^{-1}$ is continuous on $Z$.

(22) Let us consider real normed spaces $S, T, W$, a point $f$ of the real norm space of bounded linear operators from $S$ into $W$, a point $g$ of the real norm space of bounded linear operators from $T$ into $W$, and a linear operator $I$ from $S$ into $T$. Suppose

(i) $I$ is one-to-one, onto, and isometric, and

(ii) $f=g \cdot I$.

Then $\|f\|=\|g\|$. The theorem is a consequence of (9) and (7). Proof: Consider $J$ being a linear operator from $T$ into $S$ such that $J=I^{-1}$ and 
$J$ is one-to-one, onto, and isometric. Reconsider $g_{0}=g$ as a Lipschitzian linear operator from $T$ into $W$. Reconsider $g_{3}=g \cdot I$ as a Lipschitzian linear operator from $S$ into $W$. For every element $x, x \in\left\{\left\|g_{0}(t)\right\|\right.$, where $t$ is a vector of $T:\|t\| \leqslant 1\}$ iff $x \in\left\{\left\|g_{3}(w)\right\|\right.$, where $w$ is a vector of $S:\|w\| \leqslant 1\}$ by $[4,(13),(35)]$.

Let us consider $S$ and $T$. One can verify that every linear operator from $S$ into $T$ which is isometric is also Lipschitzian.

\section{Isometric Differentiable Functions on Real Normed Space}

Let us consider a real norm space sequence $G$, a real normed space $F$, a set $i$, partial functions $f, g$ from $\prod G$ to $F$, and a subset $X$ of $\prod G$. Now we state the propositions:

(23) Suppose $X$ is open and $i \in \operatorname{dom} G$ and $f$ is partially differentiable on $X$ w.r.t. $i$ and $g$ is partially differentiable on $X$ w.r.t. $i$. Then

(i) $f+g$ is partially differentiable on $X$ w.r.t. $i$, and

(ii) $(f+g) \uparrow^{i} X=\left(f \uparrow^{i} X\right)+\left(g \uparrow^{i} X\right)$.

(24) Suppose $X$ is open and $i \in \operatorname{dom} G$ and $f$ is partially differentiable on $X$ w.r.t. $i$ and $g$ is partially differentiable on $X$ w.r.t. $i$. Then

(i) $f-g$ is partially differentiable on $X$ w.r.t. $i$, and

(ii) $(f-g) \uparrow^{i} X=\left(f \uparrow^{i} X\right)-\left(g \uparrow^{i} X\right)$.

Now we state the propositions:

(25) Let us consider a real norm space sequence $G$, a real normed space $F$, a set $i$, a partial function $f$ from $\prod G$ to $F$, a real number $r$, and a subset $X$ of $\prod G$. Suppose

(i) $X$ is open, and

(ii) $i \in \operatorname{dom} G$, and

(iii) $f$ is partially differentiable on $X$ w.r.t. $i$.

Then

(iv) $r \cdot f$ is partially differentiable on $X$ w.r.t. $i$, and

(v) $r \cdot f \uparrow^{i} X=r \cdot\left(f \uparrow^{i} X\right)$.

Proof: Set $h=r \cdot f$. For every point $x$ of $\prod G$ such that $x \in X$ holds $h$ is partially differentiable in $x$ w.r.t. $i$ and partdiff $(h, x, i)=r \cdot \operatorname{partdiff}(f, x, i)$ by [19, (24), (30)]. Set $f_{3}=f \uparrow^{i} X$. For every point $x$ of $\prod G$ such that $x \in X$ holds $\left(r \cdot f_{3}\right)_{x}=\operatorname{partdiff}(h, x, i)$.

(26) Let us consider real normed spaces $S, T$, a Lipschitzian linear operator $L$ from $S$ into $T$, and a point $x_{0}$ of $S$. Then 
(i) $L$ is differentiable in $x_{0}$, and

(ii) $L^{\prime}\left(x_{0}\right)=L$.

The theorem is a consequence of (2). Proof: Reconsider $L_{0}=L$ as a point of the real norm space of bounded linear operators from $S$ into $T$. Reconsider $R=$ (the carrier of $S) \longmapsto 0_{T}$ as a partial function from $S$ to $T$. Set $N=$ the neighbourhood of $x_{0}$. For every point $x$ of $S$ such that $x \in N$ holds $L_{0 x}-L_{0 x_{0}}=L\left(x-x_{0}\right)+R_{x-x_{0}}$ by [20, (7)], [21, (4)].

(27) Let us consider a partial function $f$ from $T$ to $W$, a Lipschitzian linear operator $I$ from $S$ into $T$, and a point $I_{0}$ of the real norm space of bounded linear operators from $S$ into $T$. Suppose $I_{0}=I$. Let us consider a point $x$ of $S$. Suppose $f$ is differentiable in $I(x)$. Then

(i) $f \cdot I$ is differentiable in $x$, and

(ii) $(f \cdot I)^{\prime}(x)=f^{\prime}(I(x)) \cdot I_{0}$.

The theorem is a consequence of $(26)$.

(28) Let us consider a partial function $f$ from $T$ to $W$ and a linear operator $I$ from $S$ into $T$. Suppose

(i) $I$ is one-to-one and onto, and

(ii) $I$ is isometric.

Let us consider a point $x$ of $S$. Then $f \cdot I$ is differentiable in $x$ if and only if $f$ is differentiable in $I(x)$. The theorem is a consequence of (9), (26), and (27).

(29) Let us consider a partial function $f$ from $T$ to $W$, a linear operator $I$ from $S$ into $T$, and a set $X$. Suppose

(i) $X \subseteq$ the carrier of $T$, and

(ii) $I$ is one-to-one and onto, and

(iii) $I$ is isometric.

Then $f$ is differentiable on $X$ if and only if $f \cdot I$ is differentiable on $I^{-1}(X)$. The theorem is a consequence of (28) and (1). Proof: For every point $y$ of $T$ such that $y \in X$ holds $f\lceil X$ is differentiable in $y$ by [5, (113)].

(30) Let us consider real normed spaces $X, Y$, a partial function $f$ from $\Pi\langle X$, $Y\rangle$ to $W$, and a subset $D$ of $\prod\langle X, Y\rangle$. Suppose $f$ is differentiable on $D$. Let us consider a point $z$ of $\prod\langle X, Y\rangle$. Suppose $z \in \operatorname{dom} f_{\lceil D}^{\prime}$. Then $f_{\lceil D}^{\prime}(z)=$ $\left((f \cdot \operatorname{IsoCPNrSP}(X, Y))_{\uparrow(\operatorname{IsoCPNrSP}(X, Y))^{-1}(D)}^{\prime}\right)_{(\operatorname{IsoCPNrSP}(X, Y))^{-1}(z)^{*}}$

(IsoCPNrSP $(X, Y))^{-1}$. The theorem is a consequence of (17), (29), and (27). Proof: Set $I=\operatorname{IsoCPNrSP}(X, Y)$. Set $J=(\operatorname{IsoCPNrSP}(X, Y))^{-1}$. Set $g=f \cdot I$. Set $E=I^{-1}(D)$. For every point $z$ of $\prod\langle X, Y\rangle$ such that $z \in \operatorname{dom} f_{\uparrow D}^{\prime}$ holds $f_{\uparrow D}^{\prime}(z)=\left(g_{\uparrow E}^{\prime}\right)_{J(z)} \cdot I^{-1}$ by [10, (31)], [5, (113)], [23, $(36)]$. 
(31) Let us consider real normed spaces $X, Y$, a partial function $f$ from $X \times$ $Y$ to $W$, and a subset $D$ of $X \times Y$. Suppose $f$ is differentiable on $D$. Let us consider a point $z$ of $X \times Y$. Suppose $z \in \operatorname{dom} f_{\lceil D}^{\prime}$. Then $f_{\lceil D}^{\prime}(z)=$ $\left(\left(f \cdot(\operatorname{IsoCPNrSP}(X, Y))^{-1}\right)_{\uparrow\left((\operatorname{IsoCPNrSP}(X, Y))^{-1}\right)^{-1}(D)}^{\prime}\right)_{(\operatorname{IsoCPNrSP}(X, Y))(z)^{*}}$. $\left((\operatorname{IsoCPNrSP}(X, Y))^{-1}\right)^{-1}$. The theorem is a consequence of (18), (19), (17), (29), and (27). Proof: Set $I=(\operatorname{IsoCPNrSP}(X, Y))^{-1}$. Set $J=$ IsoCPNrSP $(X, Y)$. Set $g=f \cdot I$. Set $E=I^{-1}(D)$. For every point $z$ of $X \times Y$ such that $z \in \operatorname{dom} f_{\lceil D}^{\prime}$ holds $f_{\uparrow D}^{\prime}(z)=\left(g_{\uparrow E}^{\prime}\right)_{J(z)} \cdot I^{-1}$ by [10, (31)], [5, (113)], [23, (36)].

(32) Let us consider real normed spaces $X, Y$ and a point $z$ of $X \times Y$. Then

(i) $\operatorname{reproj} 1(z)=(\operatorname{IsoCPNrSP}(X, Y))^{-1} \cdot \operatorname{reproj}(1(\in \operatorname{dom}\langle X, Y\rangle)$,

$(\operatorname{IsoCPNrSP}(X, Y))(z))$, and

(ii) $\operatorname{reproj} 2(z)=(\operatorname{IsoCPNrSP}(X, Y))^{-1} \cdot \operatorname{reproj}(2(\in \operatorname{dom}\langle X, Y\rangle)$, $(\operatorname{IsoCPNrSP}(X, Y))(z))$.

The theorem is a consequence of (18).

Let $X, Y$ be real normed spaces and $z$ be a point of $X \times Y$. Let us note that the functor $z_{1}$ yields a point of $X$. One can verify that the functor $z_{2}$ yields a point of $Y$. Let $X, Y, W$ be real normed spaces. Let $f$ be a partial function from $X \times Y$ to $W$. We say that $f$ is partially differentiable in $z$ w.r.t. 1 if and only if (Def. 4) $f \cdot \operatorname{reproj1}(z)$ is differentiable in $z_{\mathbf{1}}$.

We say that $f$ is partially differentiable in $z$ w.r.t. 2 if and only if (Def. 5) $f \cdot \operatorname{reproj} 2(z)$ is differentiable in $z_{\mathbf{2}}$.

Now we state the propositions:

(33) Let us consider real normed spaces $X, Y$ and a point $z$ of $X \times Y$. Then

(i) $z_{1}=$ the projection onto $1(\in \operatorname{dom}\langle X, Y\rangle)((\operatorname{IsoCPNrSP}(X, Y))(z))$, and

(ii) $z_{\mathbf{2}}=$ the projection onto $2(\in \operatorname{dom}\langle X, Y\rangle)((\operatorname{IsoCPNrSP}(X, Y))(z))$.

(34) Let us consider real normed spaces $X, Y, W$, a point $z$ of $X \times Y$, and a partial function $f$ from $X \times Y$ to $W$. Then

(i) $f$ is partially differentiable in $z$ w.r.t. 1 iff $f \cdot(\operatorname{IsoCPNrSP}(X, Y))^{-1}$ is partially differentiable in $(\operatorname{IsoCPNrSP}(X, Y))(z)$ w.r.t. 1 , and

(ii) $f$ is partially differentiable in $z$ w.r.t. 2 iff $f \cdot(\operatorname{IsoCPNrSP}(X, Y))^{-1}$ is partially differentiable in $(\operatorname{IsoCPNrSP}(X, Y))(z)$ w.r.t. 2.

The theorem is a consequence of (32) and (33).

Let $X, Y, W$ be real normed spaces, $z$ be a point of $X \times Y$, and $f$ be a partial function from $X \times Y$ to $W$. The functor partdiff $(f, z)$ w.r.t. 1 yielding a point of the real norm space of bounded linear operators from $X$ into $W$ is defined by the term 
(Def. 6) $(f \cdot \operatorname{reproj1}(z))^{\prime}\left(z_{1}\right)$.

The functor partdiff $(f, z)$ w.r.t. 2 yielding a point of the real norm space of bounded linear operators from $Y$ into $W$ is defined by the term

(Def. 7$) \quad(f \cdot \operatorname{reproj} 2(z))^{\prime}\left(z_{\mathbf{2}}\right)$.

Now we state the proposition:

(35) Let us consider real normed spaces $X, Y, W$, a point $z$ of $X \times Y$, and a partial function $f$ from $X \times Y$ to $W$. Then

(i) $\operatorname{partdiff}(f, z)$ w.r.t. $1=\operatorname{partdiff}\left(f \cdot(\operatorname{IsoCPNrSP}(X, Y))^{-1}\right.$, $(\operatorname{IsoCPNrSP}(X, Y))(z), 1)$, and

(ii) $\operatorname{partdiff}(f, z)$ w.r.t. $2=\operatorname{partdiff}\left(f \cdot(\operatorname{IsoCPNrSP}(X, Y))^{-1}\right.$, $(\operatorname{IsoCPNrSP}(X, Y))(z), 2)$.

The theorem is a consequence of (32) and (33).

Let us consider real normed spaces $X, Y, W$, a point $z$ of $X \times Y$, and partial functions $f_{1}, f_{2}$ from $X \times Y$ to $W$. Now we state the propositions:

(36) Suppose $f_{1}$ is partially differentiable in $z$ w.r.t. 1 and $f_{2}$ is partially differentiable in $z$ w.r.t. 1 . Then

(i) $f_{1}+f_{2}$ is partially differentiable in $z$ w.r.t. 1 , and

(ii) $\operatorname{partdiff}\left(f_{1}+f_{2}, z\right)$ w.r.t. $1=$ $\operatorname{partdiff}\left(f_{1}, z\right)$ w.r.t. $1+\operatorname{partdiff}\left(f_{2}, z\right)$ w.r.t. 1 , and

(iii) $f_{1}-f_{2}$ is partially differentiable in $z$ w.r.t. 1 , and

(iv) $\operatorname{partdiff}\left(f_{1}-f_{2}, z\right)$ w.r.t. $1=$ $\operatorname{partdiff}\left(f_{1}, z\right)$ w.r.t. $1-\operatorname{partdiff}\left(f_{2}, z\right)$ w.r.t. 1 .

(37) Suppose $f_{1}$ is partially differentiable in $z$ w.r.t. 2 and $f_{2}$ is partially differentiable in $z$ w.r.t. 2. Then

(i) $f_{1}+f_{2}$ is partially differentiable in $z$ w.r.t. 2 , and

(ii) $\operatorname{partdiff}\left(f_{1}+f_{2}, z\right)$ w.r.t. $2=$ $\operatorname{partdiff}\left(f_{1}, z\right)$ w.r.t. $2+\operatorname{partdiff}\left(f_{2}, z\right)$ w.r.t. 2 , and

(iii) $f_{1}-f_{2}$ is partially differentiable in $z$ w.r.t. 2 , and

(iv) $\operatorname{partdiff}\left(f_{1}-f_{2}, z\right)$ w.r.t. $2=$ partdiff $\left(f_{1}, z\right)$ w.r.t. $2-\operatorname{partdiff}\left(f_{2}, z\right)$ w.r.t. 2.

Let us consider real normed spaces $X, Y, W$, a point $z$ of $X \times Y$, a real number $r$, and a partial function $f$ from $X \times Y$ to $W$. Now we state the propositions:

(38) Suppose $f$ is partially differentiable in $z$ w.r.t. 1 . Then

(i) $r \cdot f$ is partially differentiable in $z$ w.r.t. 1 , and

(ii) $\operatorname{partdiff}(r \cdot f, z)$ w.r.t. $1=r \cdot \operatorname{partdiff}(f, z)$ w.r.t. 1 .

(39) Suppose $f$ is partially differentiable in $z$ w.r.t. 2. Then 
(i) $r \cdot f$ is partially differentiable in $z$ w.r.t. 2 , and

(ii) $\operatorname{partdiff}(r \cdot f, z)$ w.r.t. $2=r \cdot \operatorname{partdiff}(f, z)$ w.r.t. 2 .

Let $X, Y, W$ be real normed spaces, $Z$ be a set, and $f$ be a partial function from $X \times Y$ to $W$. We say that $f$ is partially differentiable on $Z$ w.r.t. 1 if and only if

(Def. 8) (i) $Z \subseteq \operatorname{dom} f$, and

(ii) for every point $z$ of $X \times Y$ such that $z \in Z$ holds $f\lceil Z$ is partially differentiable in $z$ w.r.t. 1 .

We say that $f$ is partially differentiable on $Z$ w.r.t. 2 if and only if

(Def. 9) (i) $Z \subseteq \operatorname{dom} f$, and

(ii) for every point $z$ of $X \times Y$ such that $z \in Z$ holds $f\lceil Z$ is partially differentiable in $z$ w.r.t. 2 .

Now we state the proposition:

(40) Let us consider real normed spaces $X, Y, W$, a subset $Z$ of $X \times Y$, and a partial function $f$ from $X \times Y$ to $W$. Then

(i) $f$ is partially differentiable on $Z$ w.r.t. 1 iff $f \cdot(\operatorname{IsoCPNrSP}(X, Y))^{-1}$ is partially differentiable on $\left((\operatorname{IsoCPNrSP}(X, Y))^{-1}\right)^{-1}(Z)$ w.r.t. 1 , and

(ii) $f$ is partially differentiable on $Z$ w.r.t. 2 iff $f \cdot(\operatorname{IsoCPNrSP}(X, Y))^{-1}$ is partially differentiable on $\left((\operatorname{IsoCPNrSP}(X, Y))^{-1}\right)^{-1}(Z)$ w.r.t. 2 .

The theorem is a consequence of (18), (19), (17), (34), and (1). Proof: Set $I=(\operatorname{IsoCPNrSP}(X, Y))^{-1}$. Set $g=f \cdot I$. Set $E=I^{-1}(Z) . f$ is partially differentiable on $Z$ w.r.t. 1 iff $g$ is partially differentiable on $E$ w.r.t. 1 by [5. (113)], [4, (34)], [5, (38)]. $f$ is partially differentiable on $Z$ w.r.t. 2 iff $g$ is partially differentiable on $E$ w.r.t. 2 by [5, (113)], [4, (34)], [5, (38)].

Let $X, Y, W$ be real normed spaces, $Z$ be a set, and $f$ be a partial function from $X \times Y$ to $W$. Assume $f$ is partially differentiable on $Z$ w.r.t. 1. The functor $f \uparrow^{1} Z$ yielding a partial function from $X \times Y$ to the real norm space of bounded linear operators from $X$ into $W$ is defined by

(Def. 10) (i) $\operatorname{dom} i t=Z$, and

(ii) for every point $z$ of $X \times Y$ such that $z \in Z$ holds $i t_{z}=$ $\operatorname{partdiff}(f, z)$ w.r.t. 1 .

Assume $f$ is partially differentiable on $Z$ w.r.t. 2. The functor $f \uparrow^{2} Z$ yielding a partial function from $X \times Y$ to the real norm space of bounded linear operators from $Y$ into $W$ is defined by

(Def. 11) (i) dom $i t=Z$, and

(ii) for every point $z$ of $X \times Y$ such that $z \in Z$ holds $i t_{z}=$ $\operatorname{partdiff}(f, z)$ w.r.t. 2 . 
Let us consider real normed spaces $X, Y, W$, a subset $Z$ of $X \times Y$, and a partial function $f$ from $X \times Y$ to $W$. Now we state the propositions:

(41) Suppose $f$ is partially differentiable on $Z$ w.r.t. 1 . Then $f \uparrow^{1} Z=(f$. $\left.(\operatorname{IsoCPNrSP}(X, Y))^{-1} \uparrow^{1}\left((\operatorname{IsoCPNrSP}(X, Y))^{-1}\right)^{-1}(Z)\right) \cdot \operatorname{IsoCPNrSP}(X, Y)$.

(42) Suppose $f$ is partially differentiable on $Z$ w.r.t. 2. Then $f \uparrow^{2} Z=(f$. $\left.(\operatorname{IsoCPNrSP}(X, Y))^{-1} \uparrow^{2}\left((\operatorname{IsoCPNrSP}(X, Y))^{-1}\right)^{-1}(Z)\right) \cdot \operatorname{IsoCPNrSP}(X, Y)$.

(43) Suppose $Z$ is open. Then $f$ is partially differentiable on $Z$ w.r.t. 1 if and only if $Z \subseteq \operatorname{dom} f$ and for every point $x$ of $X \times Y$ such that $x \in Z$ holds $f$ is partially differentiable in $x$ w.r.t. 1 .

(44) Suppose $Z$ is open. Then $f$ is partially differentiable on $Z$ w.r.t. 2 if and only if $Z \subseteq \operatorname{dom} f$ and for every point $x$ of $X \times Y$ such that $x \in Z$ holds $f$ is partially differentiable in $x$ w.r.t. 2 .

Let us consider real normed spaces $X, Y, W$, a subset $Z$ of $X \times Y$, and partial functions $f, g$ from $X \times Y$ to $W$. Now we state the propositions:

(45) Suppose $Z$ is open and $f$ is partially differentiable on $Z$ w.r.t. 1 and $g$ is partially differentiable on $Z$ w.r.t. 1 . Then

(i) $f+g$ is partially differentiable on $Z$ w.r.t. 1 , and

(ii) $(f+g) \uparrow^{1} Z=\left(f \uparrow^{1} Z\right)+\left(g \uparrow^{1} Z\right)$.

(46) Suppose $Z$ is open and $f$ is partially differentiable on $Z$ w.r.t. 1 and $g$ is partially differentiable on $Z$ w.r.t. 1 . Then

(i) $f-g$ is partially differentiable on $Z$ w.r.t. 1 , and

(ii) $(f-g) \uparrow^{1} Z=\left(f \uparrow^{1} Z\right)-\left(g \uparrow^{1} Z\right)$.

(47) Suppose $Z$ is open and $f$ is partially differentiable on $Z$ w.r.t. 2 and $g$ is partially differentiable on $Z$ w.r.t. 2 . Then

(i) $f+g$ is partially differentiable on $Z$ w.r.t. 2 , and

(ii) $(f+g) \uparrow^{2} Z=\left(f \uparrow^{2} Z\right)+\left(g \uparrow^{2} Z\right)$.

(48) Suppose $Z$ is open and $f$ is partially differentiable on $Z$ w.r.t. 2 and $g$ is partially differentiable on $Z$ w.r.t. 2 . Then

(i) $f-g$ is partially differentiable on $Z$ w.r.t. 2 , and

(ii) $(f-g) \uparrow^{2} Z=\left(f \uparrow^{2} Z\right)-\left(g \uparrow^{2} Z\right)$.

Let us consider real normed spaces $X, Y, W$, a subset $Z$ of $X \times Y$, a real number $r$, and a partial function $f$ from $X \times Y$ to $W$. Now we state the propositions:

(49) Suppose $Z$ is open and $f$ is partially differentiable on $Z$ w.r.t. 1 . Then

(i) $r \cdot f$ is partially differentiable on $Z$ w.r.t. 1 , and

(ii) $r \cdot f \uparrow^{1} Z=r \cdot\left(f \uparrow^{1} Z\right)$.

(50) Suppose $Z$ is open and $f$ is partially differentiable on $Z$ w.r.t. 2. Then 
(i) $r \cdot f$ is partially differentiable on $Z$ w.r.t. 2 , and

(ii) $r \cdot f \uparrow^{2} Z=r \cdot\left(f \uparrow^{2} Z\right)$.

Let us consider real normed spaces $X, Y, W$, a subset $Z$ of $X \times Y$, and a partial function $f$ from $X \times Y$ to $W$. Now we state the propositions:

(51) Suppose $f$ is differentiable on $Z$. Then $f_{\uparrow Z}^{\prime}$ is continuous on $Z$ if and only if $\left(f \cdot(\operatorname{IsoCPNrSP}(X, Y))^{-1}\right)_{\uparrow\left((\operatorname{IsoCPNrSP}(X, Y))^{-1}\right)^{-1}(Z)}^{\prime}$ is continuous on $\left((\operatorname{IsoCPNrSP}(X, Y))^{-1}\right)^{-1}(Z)$.

(52) Suppose $Z$ is open. Then $f$ is partially differentiable on $Z$ w.r.t. 1 and $f$ is partially differentiable on $Z$ w.r.t. 2 and $f \uparrow^{1} Z$ is continuous on $Z$ and $f \uparrow^{2} Z$ is continuous on $Z$ if and only if $f$ is differentiable on $Z$ and $f_{\uparrow Z}^{\prime}$ is continuous on $Z$.

\section{REFERENCES}

[1] Grzegorz Bancerek. The ordinal numbers Formalized Mathematics, 1(1):91-96, 1990.

[2] Grzegorz Bancerek and Krzysztof Hryniewiecki. Segments of natural numbers and finite sequences Formalized Mathematics, 1(1):107-114, 1990.

[3] Czesław Byliński. Binary operations. Formalized Mathematics, 1(1):175-180, 1990.

[4] Czesław Byliński. Functions and their basic properties Formalized Mathematics, 1(1): 55-65, 1990.

[5] Czesław Byliński. Functions from a set to a set. Formalized Mathematics, 1(1):153-164, 1990.

[6] Czesław Byliński. Partial functions. Formalized Mathematics, 1(2):357-367, 1990.

[7] Czesław Byliński. Some basic properties of sets. Formalized Mathematics, 1(1):47-53, 1990.

[8] Agata Darmochwał. Finite sets Formalized Mathematics, 1(1):165-167, 1990.

[9] Noboru Endou, Yasunari Shidama, and Keiichi Miyajima. The product space of real normed spaces and its properties. Formalized Mathematics, 15(3):81-85, 2007. doi: $10.2478 /$ v10037-007-0010-y

[10] Hiroshi Imura, Morishige Kimura, and Yasunari Shidama. The differentiable functions on normed linear spaces. Formalized Mathematics, 12(3):321-327, 2004.

[11] Hiroshi Imura, Yuji Sakai, and Yasunari Shidama. Differentiable functions on normed linear spaces. Part II. Formalized Mathematics, 12(3):371-374, 2004.

[12] Jarosław Kotowicz. Partial functions from a domain to a domain. Formalized Mathematics, 1(4):697-702, 1990.

[13] Eugeniusz Kusak, Wojciech Leończuk, and Michał Muzalewski. Abelian groups, fields and vector spaces Formalized Mathematics, 1(2):335-342, 1990.

[14] Takaya Nishivama, Keiji Ohkubo, and Yasunari Shidama. The continuous functions on normed linear spaces. Formalized Mathematics, 12(3):269-275, 2004.

[15] Hiroyuki Okazaki, Noboru Endou, and Yasunari Shidama. Cartesian products of family of real linear spaces. Formalized Mathematics, 19(1):51-59, 2011. doi 10.2478/v10037011-0009-2.

[16] Jan Popiołek. Real normed space. Formalized Mathematics, 2(1):111-115, 1991.

[17] Laurent Schwartz. Cours d'analyse. Hermann, 1981.

[18] Yasunari Shidama. Banach space of bounded linear operators. Formalized Mathematics, 12(1):39-48, 2004.

[19] Yasunari Shidama. Differentiable functions on normed linear spaces. Formalized Mathematics, 20(1):31-40, 2012. doi 10.2478/v10037-012-0005-1.

[20] Andrzej Trybulec. Binary operations applied to functions. Formalized Mathematics, 1 (2):329-334, 1990.

[21] Wojciech A. Trybulec. Vectors in real linear space Formalized Mathematics, 1(2):291-296, 1990. 
[22] Zinaida Trybulec. Properties of subsets. Formalized Mathematics, 1(1):67-71, 1990.

[23] Edmund Woronowicz. Relations and their basic properties. Formalized Mathematics, 1 (1):73-83, 1990.

[24] Edmund Woronowicz. Relations defined on sets. Formalized Mathematics, 1(1):181-186, 1990.

[25] Hiroshi Yamazaki and Yasunari Shidama. Algebra of vector functions. Formalized Mathematics, 3(2):171-175, 1992.

Received December 31, 2013 